\title{
Virobot the Artificial Assistant Nurse for Health Monitoring, Telemedicine and Sterilization through the Internet
}

\author{
Dr. Punith Kumar M B \\ Associate Professor, Department of ECE, PES College of Engineering Mandya, Karnataka, India \\ Email:punithpes@gmail.com
}

\author{
D Manikant Amaresh Savadatti \\ Dept, of ECE, PESCE, Mandya, India \\ Email: manisavadatti@gmail.com
}

Received: 09 November 2020; Accepted: 27 November 2020; Published: 08 December 2020

\begin{abstract}
We all know that during pandemic like corona or generally in hospitals, the front-line workers such as doctors, nurses, compounder in hospitals are nearest to the patients. They all need to look after the patients without caring about themselves and hence doctors, nurses, workers have a high chance of getting contacted to the disease. And few of the medical personnel fearing for the disease and their health, they are not ready to work in the hospital. Hence to tackle this problem we have come up with a solution i.e., Virobot- The artificial nurse. The result of this project is a robot that is designed to act as a medical assistant robot which can be controlled by nurses or doctors from a distance using their smart phone or it can just use autonomous navigation to reach the patients and give them required medicines, monitor patients health and stream all the patient information wirelessly through the internet to your palm(mobile). And, it has built-in UV sterilizer lights, which sterilizes the hospital wherever it travels. Not only it can carry lightweight packages and sterilize the hospital, It can be used as a telecommunication robot ad establish communication between doctor and patient since it is a built-in FPV camera and a Bluetooth speaker. The best thing is all these features are connected to the internet hence the doctor can get data from the robot from anywhere around the world.
\end{abstract}

Index Terms: Assistant Medical robot, Autonomous robot, Blynk robot, Communication robot, Health monitor robot, Internet robot, IOT robot, Line follower, UV sterilizing robot, Wi-Fi controlled robot.

\section{Introduction}

Inspiration for Virobot the artificial nurse project was sparked by mainly three robots. The first robot is the "Moxi" robot built by Texas instrument, UV zap robots built by UVD robotics, and telepresence robots by Double robotics and Omni labs. Moxi robot is basically a robot with a robotic ARM made to carry lightweight packages. UV zap robot was built to kill germs and bacteria. A telepresence robot was made to establish communication between doctor and patient. Since all these robots work in hospitals, thought of combining all these features with some extra features and making a robot. Hence the idea to build virobot was born. And, by taking the current scenario into consideration, I thought of building robots which will both help to disinfect the place and act as a medical assistant to the doctors. .Once the idea came to my mind, it was decided to make a detailed study on this i.e., how patients are monitored and to become familiar with the environment in which the robot was supposed to work in the future. Such robots can carry medical equipment between hospital rooms and help in picking medical tools on demand.

We can categorize medical robots into two main categories. The first category consists of robots helping in medical producers fully autonomously, semi-autonomously, or being teleported by medical personnel. The second is, making social robots that will not have direct physical contact with patients or partake of medical interventions but are focused on helping people and supporting them mentally. Our robots fall in the first category. It was known that any distraction from medical procedures and any entertainment that could take a patient's attention off their health problems was beneficial. Many Psychological research projects can confirm this thesis. Also, some experiments made with robots show that interacting with robots reduces the pain and stress. Limited amounts of qualified personnel or busy relatives make it impossible to ensure that every man in need has their own assistant. That is the place where virobot can help and improve the quality of life of the elderly. For people with memory disorders, virobot can play a role of a reminder 
about medications. Tracking people, diagnosing and monitoring their vital functions are also noted as major advantages.

\section{Literature Review}

Marcin Zukowski [1] In this paper a concept of the humanoid mobile robot which is dedicated to children hospitals was presented. It was designed for two practical functions; it was a robotic assistant for medical staff and a companion for young patients. Mark Anderson [2], In this an office was made where you can disinfect your keys, phone, and other portables at the reception area's ultraviolet-sterilization stand. In cooler months, the air we breathe was cleansed of mold and bacteria in UV-sterilized heating units as well as blasted by UV fixtures in the office air ducts to eliminate viruses. Wan-Ling Chang [3], It shows that instead of a robot which completely replaces human labor, nursing home staff prefer robot assistants who fit into their working process. It learned that most appropriate functions for robots in nursing homes were helping with minor works. Shamsudeen Abubakar [4], In this paper it presents a mobile manipulator designed to serve as an assistant to nurses in patient walking and patient sitting tasks in hospital environments. Jesus Alvarez[5] , Nurse-bot it is like-a nurse made robot that monitors vital signs and keeps the information, so that the attending physician has up-to-date parameters before the consultation that allows a better follow-up of the results of the prescribed treatment made by a specialist. Ronald Marchessault [6], RoNA system in this paper provides physical assistance to nurses in a hospital ward. This robot would increase job satisfaction, reduce lifting-related injuries, and extend the years of effective service nurses could render in hospitals. Danica Mitch M. Pacis1 [8], The trends in the development of the utilization of this technology can be classified in to four: patient monitoring, healthcare information technology, intelligent assistance diagnosis, and information analysis collaboration.

\section{Methodology}

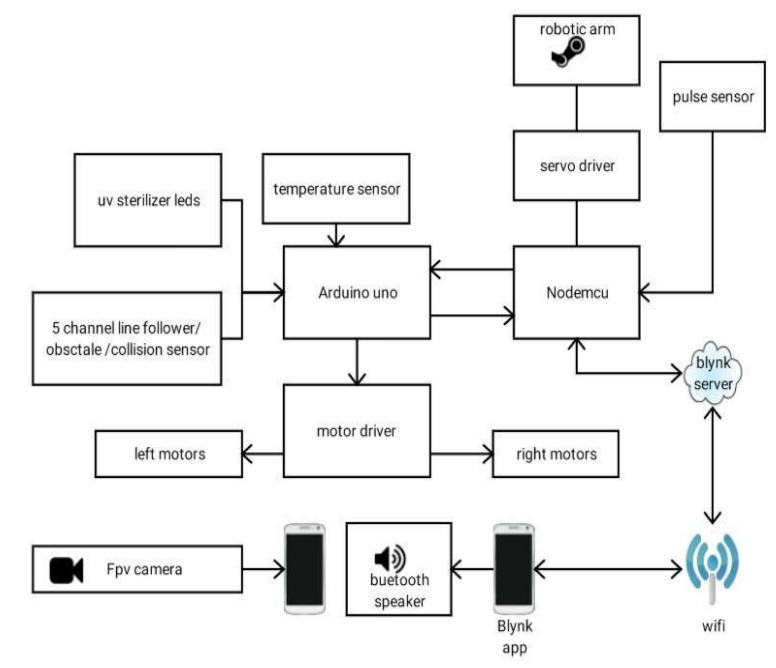

Fig. 1. Virobot-Block Diagram

Figure. 1 represents a basic working block diagram of the robot. The whole methodology can be divided into two parts.

\section{Objectives}

1) Creating advanced and cost-effective indoor navigation systems based on advanced line follow and obstacle avoidance technology in order to make the navigational capabilities of Virobot was more robust.

2) Making the artificial nurse robot which can use line following mode - The robot transverse the path according to black or white line following algorithm using a 5-channel digital line following sensor. Wireless remote control - The robot movement is controlled by Wi-Fi or internet from anywhere using mobile

3) Medical Transportation: Giving medicines and meals to patients and staff by adding a tray and a robotic ARM to our Virobot, thereby optimizing communication between doctors, hospital staff members, and patients.

4) Using our virobot to clean a room contaminated with bacteria and germs within minutes using UV-C light

5) Monitoring basic health parameters like heart rate and temperature using virobot

6) Establishing communication between doctor and patient.

7) Establishing serial communication between Arduino UNO and NodeMCU.

8) Mobile Application: The dedicated mobile application Blynk includes a virtual joystick for remote control and with a live video feed. 


\section{Feature}

$>$ Our virobot consists of a base consisting of 4 wheels run by $12 \mathrm{v} 1000 \mathrm{rpm}$ Dc Motors

$>$ The motors are controlled by the motor driver.

$>$ The base also consists of a line sensor which helps to make line-following robots.

$>$ The line sensor input is processed by Arduino board and sends signals to motors via motor driver

$>$ The virobot consists of a plate or a holder to keep tablets, medicines, etc

$>$ The virobot as robotic an ARM of 4 degrees of freedom which is controlled by NodeMCU via servo driver

$>$ The virobot consists of UV light sterilization leads all over the body, tray, and at the base.

$>$ Virobot's ARM as a camera mounted to its robotic ARM.

$>$ Since it is mounted to the robotic ARM the doctor can get surrounding video by taking robotic ARMs 4 .

$>$ It has a temperature of the sensor module, which can be used to detect the temp of the patient.

$>$ Our virobot can be used in places like airports, railway stations, hospitals for

$>$ Checking on the patients instead of employing humans.

$>$ It as human colloid detection and obstacle the sensor which helps it to stop hitting humans or objects accidentally

Hardware consisting of Arduino UNO, NodeMCU, Robotic ARM, Dc motors, Motor driver, Line following sensor, 12v battery, LM35 temperature sensor, Wi-Fi camera, UV LEDs

Arduino UNO: The Arduino UNO as a microcontroller ATmega328P as shown in figure 2. Arduino is an opensource platform and its simple design and using ability makes it ideal for hobbyists and professionals. The Arduino UNO has 14 digital I/o pins (6 can be used as PWM outputs), 6 analog input pins, a $16 \mathrm{MHz}$ crystal oscillator, a USB connection port, a dc power jack, an ICSP header, and also a reset button

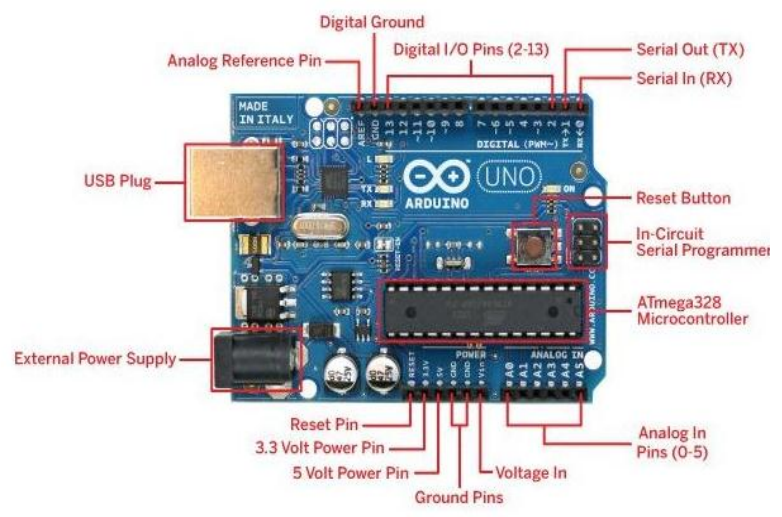

Fig.2. Arduino Microcontroller

NodeMCU: NodeMCU is an open-source development board and firmware based on the widely used ESP8266 12E Wi-Fi module as shown in figure 3. It allows you to program the ESP8266 Wi-Fi module with powerful LUA programming language or Arduino IDE. Arduino-like hardware I/O, USB-TTL included, it has 10 GPIOs D0-D10 with PWM functionality, IIC, and SPI communication, 1-Wire, and ADC at A0, etc. it is an all in one board. It is an Eventdriven API for network applications with PCB antenna.

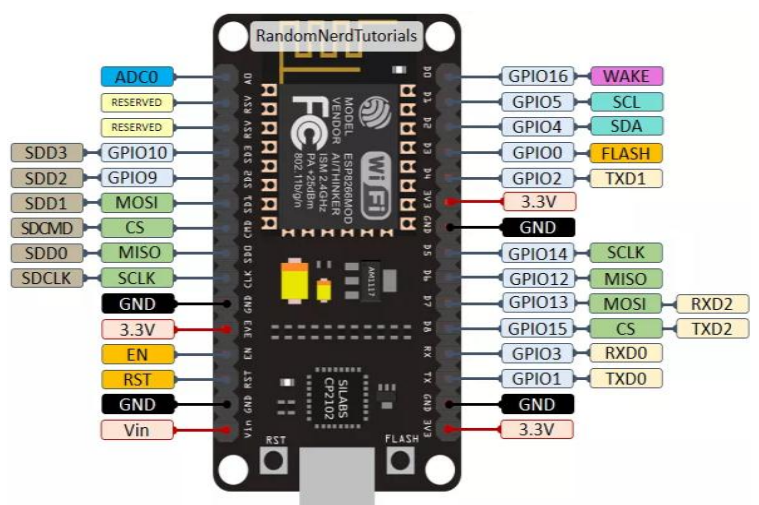

Fig. 3. NodeMCU 
Robot ARM: It is a programmable mechanical ARM as shown in figure 4, which functions the same as that of a human ARM. The links of a robotic ARM are connected using servo motors allowing either translational or rotational motion. The robotic ARM has three parts namely base, elbow, and gripper. Each part has one degree of freedom which means it will have 4 movements. Servo Motor will be used to move the robot ARM and will be located at every joint of the robot ARM.

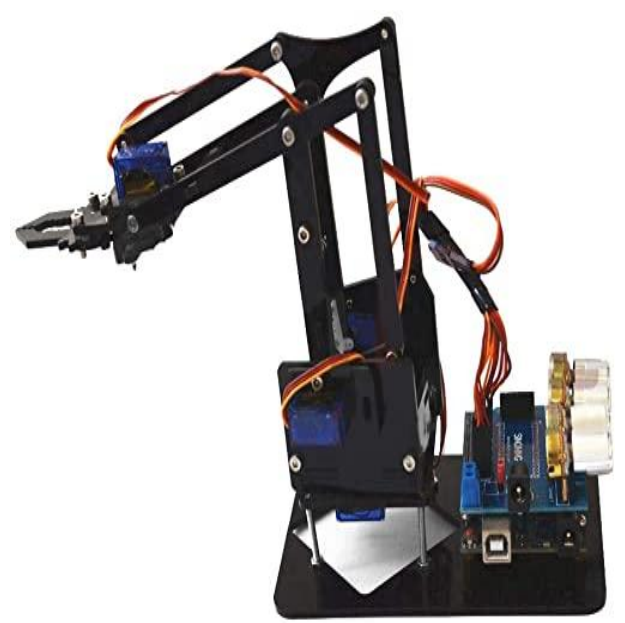

Fig. 4. Robot ARM

Motors: Permanent-magnet direct-current (PMDC). These motors as shown in figure 5 are easy to use because both their direction and speed can be controlled, direction by polarity, and speed by voltage. DC Motors are mostly used in robotics as shown in figure 5 because of their small size and more energy output, they are best for driving the mechanical assemblies.

$>$ 1000RPM 12V DC motors with Gearbox

$>6 \mathrm{~mm}$ shaft diameter with internal hole

$>125 \mathrm{gm}$ weight

$>$ Stall Torque $=0.5 \mathrm{kgcm}$ torque

$>$ No-load current $=60 \mathrm{~mA}(\mathrm{Max})$

$>$ Load current $=300 \mathrm{~mA}($ Max $)$

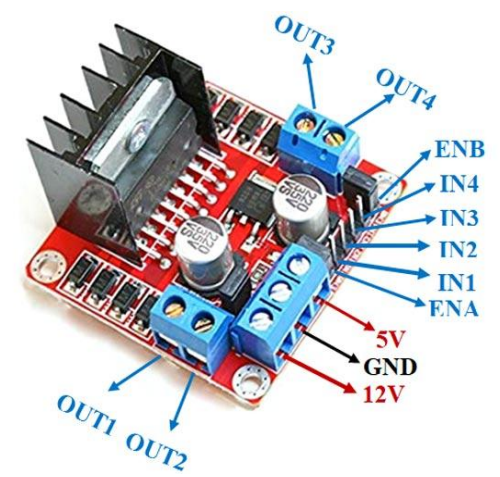

Fig. 5. Motor Driver

Motor Driver: L298N Module is a motor driver module for driving DC and Stepper Motors. This module has an L298 motor driver IC and a 78M05 5V regulator. L298N Module can control up to four DC motors, or just two DC motors with directional and speed control

Driver Chip: Double H Bridge L298N

$>$ Motor Supply Voltage (Maximum): 46V

$>$ Motor Supply Current (Maximum): 2A

$>$ Driver Voltage: $5-35 \mathrm{~V}$

$>$ Logical Current:0-36Ma

Maximum Power (W): 25W 
Line Following Sensor: As shown in figure 6 it has an Integrated 5-way tracking sensor with high sensitivity for low environmental impact. It integrates a barrier infrared sensor, unblinded distance can be adjusted by using a rheostat. It has a specially designed touch detection sensor. All the output signal to a digital signal convenient for the microcontroller. It has all the sensor output status LED lights as an indicator for easy debugging. It supports voltage of $3.0-5.5 \mathrm{v}$ to meet most system requirements. The detecting distance is about $0-4 \mathrm{~cm}$ Input voltage: $3.0-5.5 \mathrm{~V}$

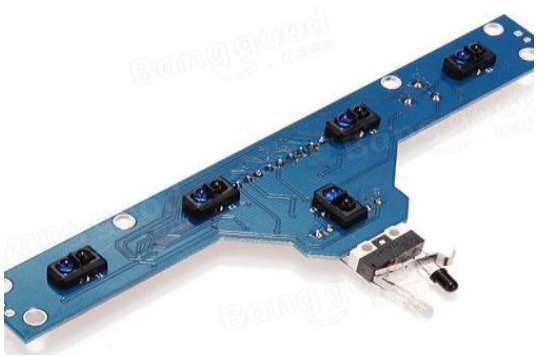

Fig. 6. Line Sensor

Battery: Battery pack Capacity: 2000mah, Battery Type: Rechargeable Li-on battery. InputVoltage:12.6V, Output voltage: 11.1 - 12.6v DC , Output Current: About 1-3A, Energy - Nominal Voltage: 11.1V - Output, Voltage Range: $11.1 \mathrm{v}-12.6$ by test conditions

Temperature Sensor: LM35 is a precession IC Temperature sensor, whose output voltage changes, based on the temperature around it. It can be used to measure temperature anywhere between $-55^{\circ} \mathrm{C}$ to $150^{\circ} \mathrm{C}$. It can be connected with any Microcontroller.

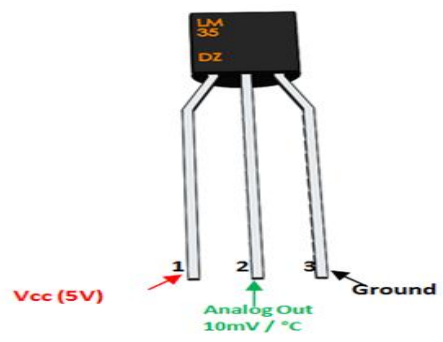

Fig. 7. Temperature Sensor

Minimum and Maximum Input Voltage of the sensor is $35 \mathrm{~V}$ and $-2 \mathrm{~V}$, respectively. Measuring range is $-55^{\circ} \mathrm{C}$ to $150^{\circ} \mathrm{C}$, Output voltage is directly proportional (Linear) to temperature, There will be a rise of $10 \mathrm{mV}$ for every $1^{\circ} \mathrm{C}$ rise in temperature. $\pm 0.5^{\circ} \mathrm{C}$ Accuracy, Drain current is less than $60 \mathrm{uA}$

Wi-Fi FPV Camera: These cameras as shown in figure 8 have an integrated Wi-Fi chip through which they stream the live video feed to their mobile phone. It weighs around $200 \mathrm{gm}$. Its dimension is $21 \times 16 \times 7 \mathrm{~cm}$. We can download an app from the play store and the camera will connect to mobile Wi-Fi and streams it to the mobile app. Through which you can record video and capture images

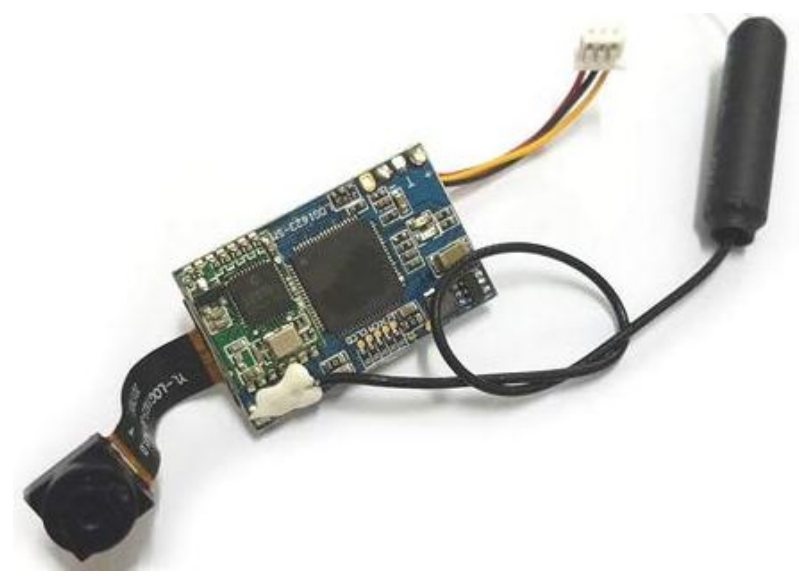

Fig. 8. Wi-Fi FPV Camera 
UV Sterilizing LEDs: In particular, the wavelength of $264 \mathrm{~nm}$ is incredibly impressive at killing germs, viruses, and bacteria. Fortunately, UV-C radiation can pass through air without creating ozone, so UV-C lamps can be used in the air to disinfect surfaces. These are $5 \mathrm{~mm}$ Ultraviolet LEDs with a voltage drop of $3 \mathrm{~V}-3.6 \mathrm{~V}$

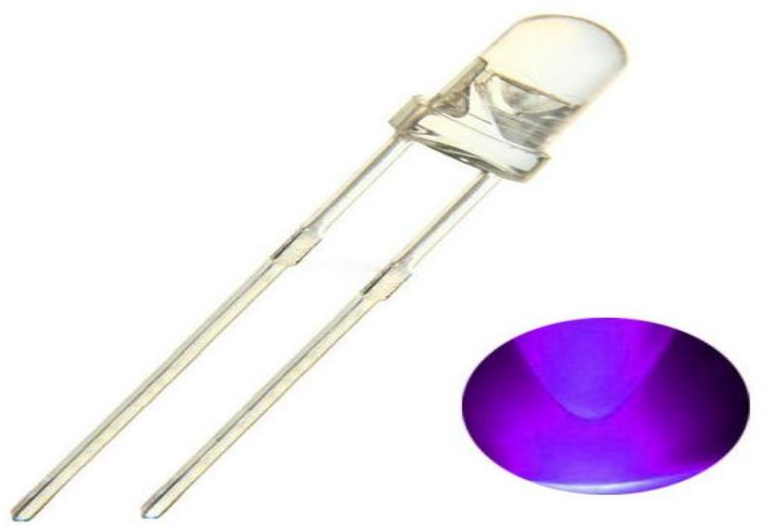

Fig. 9. UV Sterilizing LEDs

Mechanism: The base is made up of high strength steel to support the weight of the whole robot. High torque four $12 \mathrm{v}$ DC motor is attached to the base for movement. All the four motors are connected to an $1298 \mathrm{n}$ motor driver. The motor driver can be used to control the speed and direction of the robot. The motor driver is connected to Arduino UNO. There is a 5-channel digital line following the sensor with an obstacle sensor and collision detector in front of the base. The robot has two modes one is line following mode and the second is wireless control. When the robot is in line following mod the line following sensor sends a signal to the Arduino UNO. Arduino by using the line following algorithm sends signals to the motor driver for the movement of the robot. When the robot is in wireless mode the NodeMCU sends signals to the Arduino via serial communication, the Arduino reads those signals and sends the signal to the motor driver which control the motors.

Line Following Mode: The IR sensor consists of an IR LED as shown in figure 10 and an IR Photodiode. The IR light emitted will strike the surface and is reflected to the IR Photodiode. The photodiode then generates an output voltage proportional to the reflectance level of the surface. In the case of our sensor, an integrated circuit at the module generates an output digital signal (HIGH: Dark; LOW: Light).

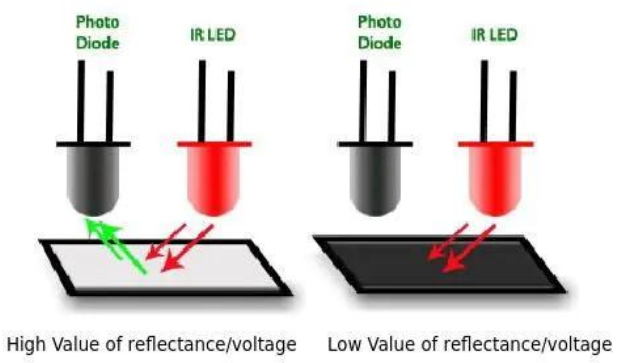

Fig. 10. IR LED

The array of 5 sensors is mounted such that only one sensor is centered with relation to the black line, only that specific sensor will produce a HIGH. The space between sensors should be calculated to allow that 2 sensors can cover the full-width edge of the black line simultaneously, producing also a LOW on both sensors.
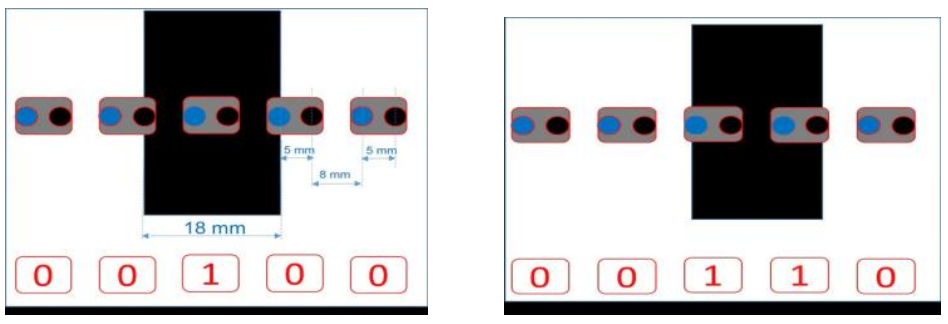

Fig. 11. Line follower logic 
Let us consider an optimum condition when the robot is centered as shown in table 1 having the line just below the "middle sensor". The output of the array will be 00100 . If the robot starts to move left the line moves right so sensor output is 00010, hence we need to make the robot turn right till its center sensor is on the backline. If the robot starts to move to the right the line starts to move left so sensor output is 01000 , hence we need to move the robot left till its center sensor is on the blackline.

Table 1. Sensor output and robot direction

\begin{tabular}{|c|c|}
\hline Sensor Output & Robot Direction \\
\hline 00001 & right \\
\hline 00011 & right \\
\hline 00010 & right \\
\hline 00110 & left \\
\hline 00100 & straight \\
\hline 01100 & left \\
\hline 01000 & left \\
\hline 11000 & left \\
\hline 100000 & left \\
\hline
\end{tabular}

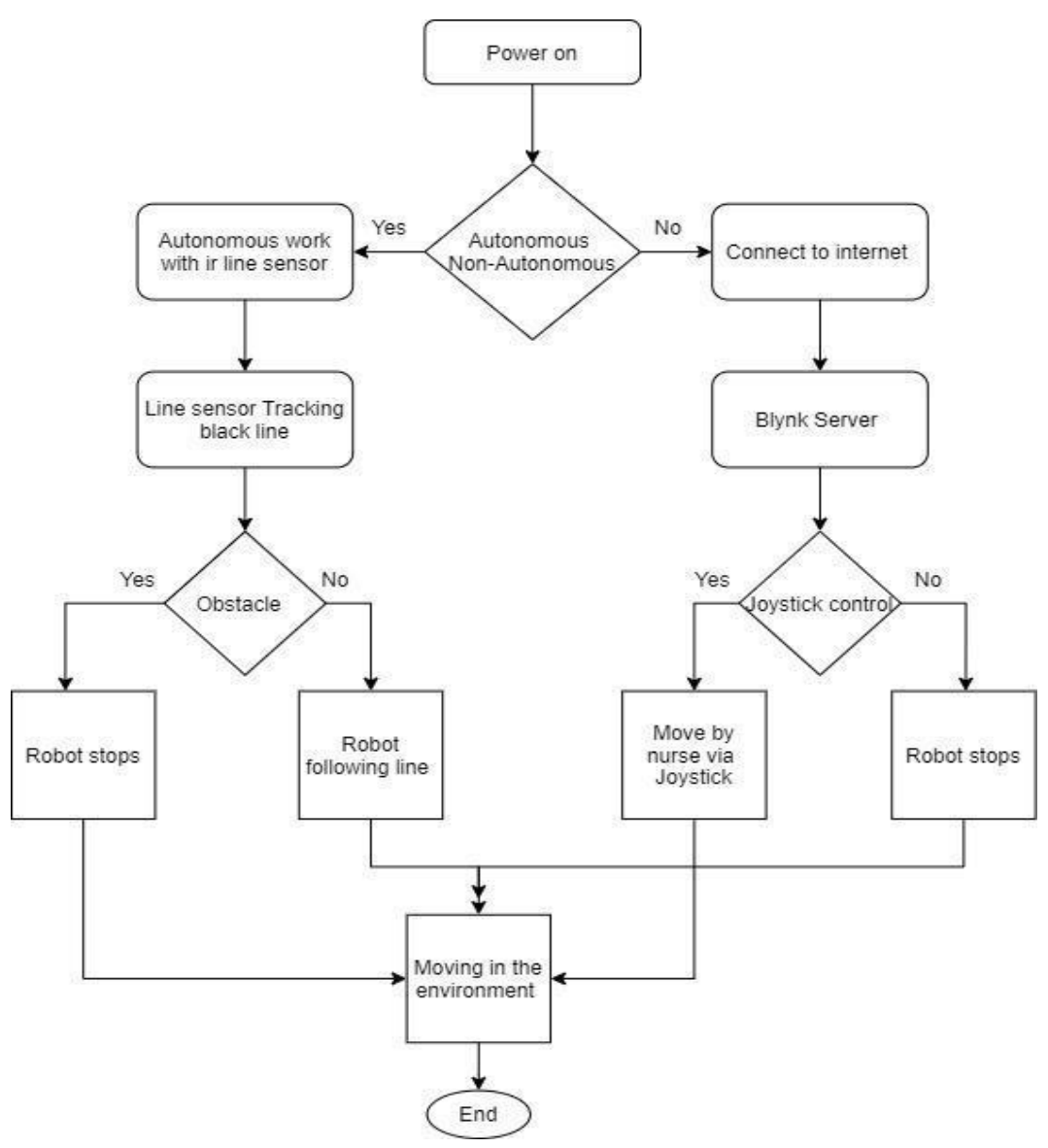

Fig. 12. Movement control working block diagram

Wireless Control: When the user selects the wireless mode the robot switches from the line following to wireless control mode. The user controls the robot using a joystick present in the Blynk app. When the user moves the joystick forward the app connected to the internet sends a signal to the Blynk server, the NodeMCU connected to the Blynk server reads the signal and sends the signal to Arduino via serial communication. Now Arduino sends the signals to the motor driver to move the robot forward. Similarly, the robot moves in all directions depending on user signals. In the second tab, it is a slider for ARM control, each slider is to control each joint of the robotic ARM. In the third tab, we have a sensor gauge that shows heart rate BPM and temperature of a patient, depending on the virtual pin chosen it shows pulse and LM35 sensor reading. There are buttons which you can see in the first image. If both buttons are off $(00)$ UV sterilizing LEDs turn on and the robot starts sterilizing itself and medical equipment. If the combination is $(10)$ then the robot is in 
wireless control mode. If the combination is $\left(\begin{array}{ll}0 & 1\end{array}\right)$ then the robot is in autonomous line following mode. When the combination is (11) the robot starts streaming the sensor measurements to the Blynk app

Virobot Movement Control Working: Figure 12 explains you the basic movement control mechanism of the Virobot. The whole block diagram is explained here. As soon as the power turns on robot boots up. The Arduino UNO and NodeMCU will power up and the serial communication between the starts as shown in figure 12. Since there are two microcontrollers both process data parallelly. First, let me start by explaining the Arduino process, the robot checks if there is an obstacle if there is an obstacle detected the robot stops and does nothing. If no obstacle is detected the robot checks for different logical conditions. first the overflow checks if the robot is given logical 01 to activate the autonomous mode. if this condition is not satisfied the robot moves on to the next condition that is it checks if the condition is 10 if the condition is satisfied the robot will activate its wireless mode and the robot can be controlled using the Blynk app joystick through the internet. If this condition is false the Arduino checks for another condition i.e,00 if this condition is met the robot turns on the UV LEDs if the condition is false the robot turns off the LEDs. Now let me explain the parallel operation taking place in NodeMCU. As soon as the NodeMCU connects to the Blynk server NodeMCU starts reading data from the blank server and sends the required data to Arduino via serial communication. when the slider is moved in the Blynk app the NodeMCU receives the command moves the robotic ARM. If no command is received NodeMCU checks if there is logical 11 coming from the server if the data arrives the NodeMCU reads the data from the sensors and streams the data to the Blynk server. The Blynk server streams the data into the sensor gauge of the Blynk app and the process keeps on repeating till power is turned off.

\section{Whole Robot Code Working Flowchart:}

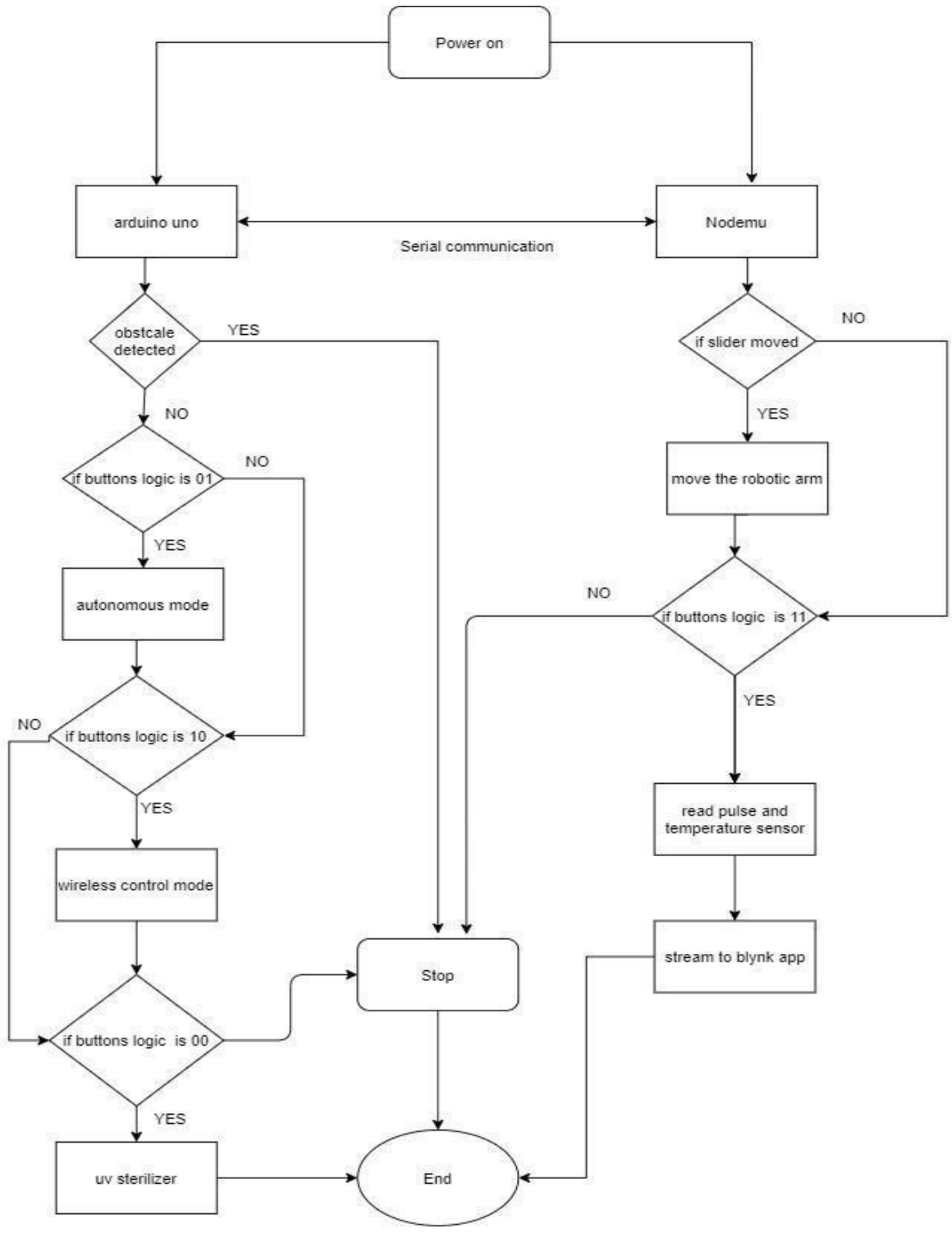

Fig. 13. Algorithm working flowchart 
Fig. 13. Explains the Algorithm of the code represented by a flow chart

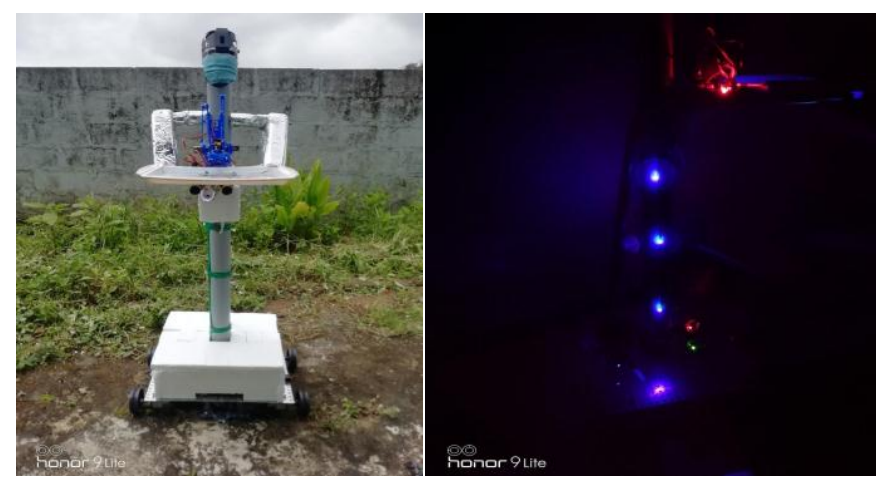

Fig. 14. Virobot Front View and UV Mode

\section{Experimental Results}

After the robot was built all its features were tested. The line following mode was tested several times and improved for every testing. The image [15.1] shows the result of virobot following the black line. Since it is using a 5channel line sensor so it can cover larger area and detect edges and curves easily, most of the time the robot was able to follow the black line without any problems. And the obstacle sensor as stopped the robot as you can see in the figure 15.2, This shows that the robot is able to reach the patient bed by autonomous mode and stop if there is obstacle on its way. Its wireless control mode would have a delay due to wireless data sending and serial communication. So, lots of efforts were made to reduce delay in data transmission to just 0.1 second per command

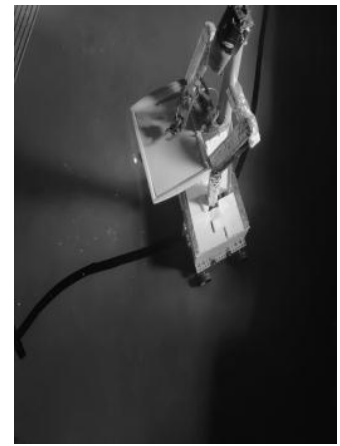

Fig. 15.1

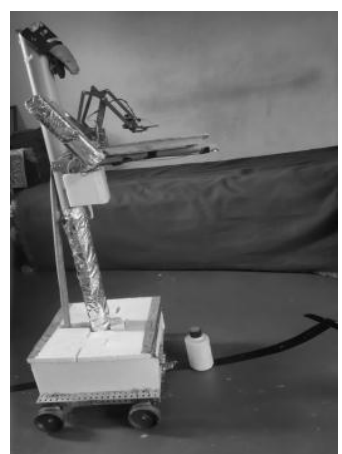

Fig. 15.2

After the robot reaches the patient by autonomous or manual mode it will use its robotic ARM to give the medicine, the figure[15.3] shows you the result of the robotic ARM picking and giving the medicine.

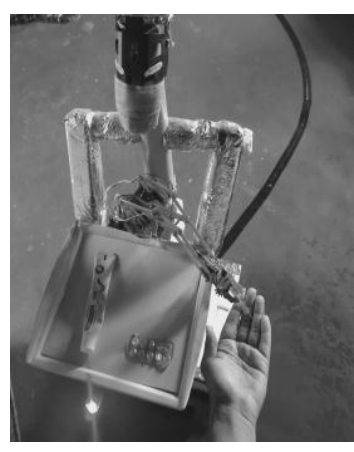

Fig. 15.3

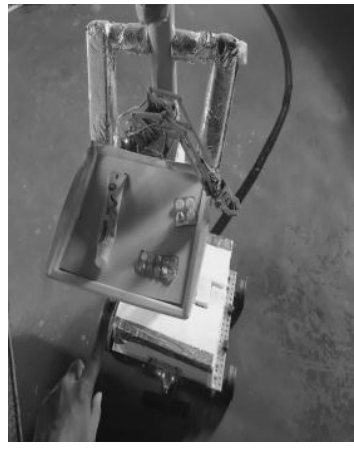

Fig.15.4

The 4 dof robot ARM was able to pick and give light weight medicines and equipment's. Patient places his finger on the sensor as shown in figure 15.4 to measure temperature and heartrate. The temperature sensor and heart rate sensor were quite accurate showing the BPM rate between 68-80 and temperature sensor was showing the temperature from $96 \mathrm{~F}-100 \mathrm{~F}$ as shown in .Hence, the robot can be now used in hospitals or any place to monitor patient's health or carry light weight packages (up to $2 \mathrm{~kg}$ ). 


\section{Conclusion}

Current Works are focused on providing autonomous navigation of the robot in an unknown, dynamic environment with moving objects. Making robots perform basic medical tasks is in development. We keep constantly working on improving human-robot interaction. The robot in its initial form, would play significant roles and would assist medical staff in some of their everyday chores.

The future work is to make the robot more interaction friendly to patients and have more accurate and efficient autonomous capabilities. Adding sensors to measure more parameters of human bodies, like o2 level, BP, ECG

In future this project can stand as a great field for conducting different science research, and testing engineering solutions. Humanoid robotic medical assistants and human assistants will be simultaneously working in one hospital.

\section{References}

[1] Yazdi, Khaoula, DARMoul, Saber \& Hajri- Gabouj, Sonia (2018), 'Intelligent product quality control and defect detection: A case study', 2018.

[2] Humanoid medical assistant and companion robot for patients Cite as: AIP Conference Proceedings 2029, 020086 (2018); Published Online: 29 October 2018 Marcin Zukowski, Krzysztof Matus, Dawid Kamienski, Krzysztof Kamil Sadowski, Kamil Guz, Miro slaw Kondratiuk, and Leszek Ambroziak

[3] Mark Anderson, ultraviolet offense: The ultraviolet offense: Germicidal UV lamps destroy vicious viruses Published in: IEEE Spectrum (Volume: 57, Issue: 10 , Oct. 2020)

[4] Wan-Ling Chang, Selma Šabanović, Potential Use of Robots in Taiwanese Nursing Homes, 2013 IEEE Xplore

[5] Shamsudeen Abubakar, Student Member IEEE, Sumit K. Das1, Member IEEE, Chris Robinson1, Student Member IEEE, Mohammed N. Saadatzi1, Member IEEE, M. Cynthia Logsdon2, Heather Mitchell2, Diane Chlebowy2, and Dan O. Popa1, Senior Member IEEE -ARNA, a Service robot for Nursing Assistance: System Overview andUser Acceptability,August 20-21, 2020

[6] Jes'us Alvarez, Guillermo Campos, Valeria Enr'iquez, Alexis Miranda, Francisco Rodriguez, Hiram Ponce, Nurse-Bot: A Robot System Applied to Medical Assistance.

[7] Ronald Marchessault, John Hu, Aaron Edsinger, Yi-Je Lim, Nick Donaldson, Mario Solano, and Aaron Solochek, An Advanced Medical Robotic System Augmenting Healthcare Capabilities - Robotic Nursing Assistant. Cite as: AIP Conference Proceedings 1933, 040009 (2018); Published Online: 13 February 2018.

[8] P. Fernandez-Marcelo, "Telehealth and eMedicine," Presentation, National Telehealth Center, 2013.

[9] E. Dinya and T. Tóth, "Health Informatics: eHEALTH and TELEMEDICINE," Presentation, Semmelweis University Institute of Health Informatics, 2013.

[10] World Health Organization, "2010 Opportunities and developments Report on the second global survey on eHealth Global Observatory for eHealth series -Volume 2 TELEMEDICINE in Member States WHO Library Cataloguing-inPublication Data," Report, WHO Press, 2010.

[11] C. S. Pattichis, E. Kyriacou, S. Voskarides, M. S. Pattichis, R. Istepanian, and C. N. Schizas, IEEE Antennas Propag. Mag. 44(2), pp. 143-153 (2002).

[12] T. Chea, "Telepresence robots let medical specialists 'beam' into remote hospitals | Lubbock Online | Lubbock AvalancheJournal,” 2013-11-17, 2013. [Online]. Available: http://lubbockonline.com/filed-online/2013-11- 17/telepresence-robots-letmedical-specialists-beam-remote-hospitals. [Accessed: 24-Jun-2017].

[13] iRobot Corp., "FDA Clears First Autonomous Telemedicine Robot for Hospitals | Business Wire." [Online].Available:http://www.businesswire.com/news/home/20130124005134/en/FDA-Clears-AutonomousTelemedicineRobot-Hospitals. [Accessed: 24-Jun-2017].

[14] S. Choudary, "Dr Rho - Medical Telepresence Robot :: Create the Future Design Contest," 2014-07-2, 2014. [Online]. Available:https://contest.techbriefs.com/2014/entries/medical/5078.

\section{Authors' Profiles}

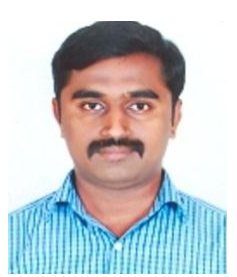

Dr. Punith Kumar M B was born in Mandya, India, in 1985. He received the B.E. Degree in Electronics and Communication Engineering from The National Institute of Engineering, Mysore in 2007, and the M.Tech in VLSI Design and Embedded Systems from PES College of Engineering, Mandya under the The Visvesvaraya Technological University (VTU), Belgaum in 2010 and Ph.D. degrees in Electronics from the University of Mysore (UoM), Mysore, India, in 2017.

In 2007, he joined the Department of Electronics and Communication Engineering as a Lecturer in JVIT, Bidadi, Bangalore. In 2010, he joined the Department of Electronics and Communication Engineering as a Assistant Professor in BGS Institute of Technology, BG Nagar, Mandya. In 2017, he joined the Department of Electronics and Communication Engineering as a Associate Professor in PES College of Engineering Mandya. His current research interests include image processing, video processing, video shot detection etc. Dr Punith Kumar M B is a Life Member of the Indian Society for Technical Education (ISTE) and Associate Member of the Institution of Engineers (AMIE), Member of IEEE. He was the Judge, Chairperson and Review member for the National and International Conference. 
D Manikant Amaresh Savadatti student of the Dept, of ECE, PESCE, Mandya, India. He is interested in carryout the projects based on the image processing and Robot, IOT.

How to cite this paper: Punith Kumar M B, D Manikant Amaresh Savadatti, " Virobot the Artificial Assistant Nurse for Health Monitoring, Telemedicine and Sterilization through the Internet", International Journal of Wireless and Microwave Technologies(IJWMT), Vol.10, No.6, pp. 16-26, 2020.DOI: 10.5815/ijwmt.2020.06.03 